\title{
INSTITUCIONALIDAD Y TENDENCIAS DE LA GESTIÓN CULTURAL MUNICIPAL EN JALISCO, MÉXICO
}

\author{
José Luis MARISCAL OROZCO \\ Universidad de Guadalajara (México) \\ mariscal@udgvirtual.udg.mx
}

\section{INSTITUTIONALITY AND TRENDS IN MUNICIPAL CULTURAL MANAGEMENT IN JALISCO, MEXICO}

Resumen: En las últimas dos décadas, ha habido un crecimiento sostenido de la infraestructura y los servicios culturales en los municipios de Jalisco, sin embargo éste no va acompañado de un fortalecimiento institucional y una profesionalización en la gestión cultural municipal. En ese sentido hay una diversidad en las formas institucionales desde donde se diseña y opera la acción cultural, que aunado a los perfiles de los responsables de dirigirla, la gestión cultural municipal tiende hacia la programación de actividades de corte artístico y patrimonialista dejando de lado la visión integral y estratégica de la cultura como parte del desarrollo social de los municipios.

Abstract: There has been a steady growth in infrastructure and cultural services in the municipalities of Jalisco in the last two decades. However, it is not accompanied by institutional strengthening nor professionalization of municipal cultural management. Hence there is much diversity in institutional ways of designing and operating cultural actions, and this, added to the profiles of those responsible for directing them, makes municipal cultural management to become programming of artistic and patrimony-related activities, setting aside an integral and strategic vision of culture as part of the social development of municipalities.

Palabras clave: Gestión cultural. Política cultural. Gobierno local. Institucionalidad cultural Cultural management. Cultural policy. Local government. Cultural institutions 


\section{Introducción}

De acuerdo a los juristas, el municipio es la célula básica del Estado, ya que articula cuestiones territoriales, gubernamentales y administrativas en una misma figura jurídico política que tiene ya una larga trayectoria prehispánica y liberal en nuestro país (Vicencio, 1986; Riva Palacio, 1985). Desde una perspectiva federalista, se visualiza al municipio como como una "comunidad básica", o "casa grande" donde los ciudadanos, a partir de su interrelación afrontan los problemas comunes a partir del diálogo, acuerdos y puesta en marcha de acciones en común (Flores, 1985; Gutiérrez).

Sin embargo, para los gobiernos locales, no siempre es fácil atender las "problemáticas comunes" esto debido a una serie de factores externos como el centralismo, limitaciones hacendarias y presupuestales, las crisis económicas y sociales, etc.; pero también internas como la lucha por los intereses de los grupos, falta de cuadros profesionales, visión a corto plazo de las acciones, etc. En México existe una gran heterogeneidad en los municipios en cuanto a tamaño, infraestructura urbana, potencial económico, etc., que generan una desigualdad entre los ciudadanos para acceder a los servicios de salud, vivienda, laborales, legales, educativos, recreativos y por supuesto, culturales.

En el caso del Estado de Jalisco en México, la mayor concentración de los servicios se da en la Zona Metropolitana de Guadalajara que congrega al 60\% de la población, dejando el $10 \%$ para las ciudades medias y el $30 \%$ para el resto de los municipios (INEGI, 2011).

La infraestructura y servicios culturales también se concentran en la capital del estado, sin embargo en las últimas dos décadas ha habido un crecimiento sostenido de éstos en las diversas regiones de Jalisco. Se han abierto y rehabilitado casas de cultura, museos, centros culturales que pretenden dar atención a la población de los municipios jaliscienses (Secretaría de Cultura de Jalisco, 2012; Castro, 2011).

Sin embargo, el incremento de la infraestructura y servicios culturales municipales ${ }^{1}$, no siempre va acompañado de un fortalecimiento institucional que permita una sustentabilidad de los espacios ganados, pero también que cumplan su propósito de satisfacer las necesidades culturales de las comunidades donde están ubicados. En el mismo tenor, el diseño y contenido de la acción cultural municipal no necesariamente responde a visión estratégica del desarrollo cultural que se desea tener, sobre todo en concordancia con una gestión cultural contemporánea que contempla la articulación de diversos aspectos sociales, económicos y ambientales desde el espacio local desde un modelo de corresponsabilidad.

Así pues, este artículo tiene como propósito hacer un acercamiento a la gestión cultural municipal en el Estado de Jalisco (México) a partir de tres elementos de observación: 1) Las formas de institucionalidad cultural que existen y se han venido configurando en los últimos diez años. 2) los perfiles de los agentes culturales responsables de la acción cultural a nivel municipal y 3) las principales acciones culturales que realizan las estancias gubernamentales analizadas a la luz de la agenda 21 para la cultura.

Este documento expone parte de la información recolectada de una investigación ${ }^{2}$ que tuvo como objeto de estudio el programa Jalisco en la Cultura que operó la Dirección General de Vinculación Cultural de la Secretaría de Cultura entre 2007-2012. ${ }^{3}$ Cabe men-

\footnotetext{
1 Hay que hacer la aclaración de que se debe distinguir entre la infraestructura y servicios que ofrece el gobierno del estado y los que ofrecen los gobiernos municipales, este artículo considera como parte de su análisis y exposición sólo a los segundos.

2 La metodología utilizada contempló la implementación de un cuestionario mixto, revisión documental, etnografía y entrevistas individuales y grupales. Más detalles de la metodología se irán presentando en el transcurso del texto.

3 Este programa tenía cuatro ejes de acción: 1) infraestructura cultural, enfocado a crear y fortalecer museos, bibliotecas y casa de cultura municipales; 2) Formación cultural, consistente en la realización de talleres artísti-
} 
cionar, que la Secretaría de Cultura de Jalisco juega un papel fundamental en la definición y operación de la política cultural en Jalisco, sin embargo, el objetivo de la investigación fue analizar los perfiles y formas en que los agentes culturales perciben y hacen la gestión cultural desde y para sus municipios. ${ }^{4}$

\section{Institucionalidad cultural en los municipios jaliscienses}

Para la gestión cultural, la institucionalidad cultural es un instrumento jurídico, político y administrativo del Estado para diseñar y ejecutar políticas culturales públicas. De acuerdo a Garretón (2008) contempla dos dimensiones: La orgánica, relacionada con las formas organizacionales que los gobiernos definen para diseñar y operar la acción cultural de intervención directa; y la normativa, que refiere al conjunto de leyes y normas en el ámbito de la cultura.

La institucionalidad cultural orgánica de Jalisco, existen varios organismos que son responsables de la acción cultural a nivel estatal, sin embargo las dos grandes figuras: La Secretaría de Cultura (SCJ) y el Consejo Estatal para la Cultura y las Artes (CECA). La primera es el órgano operativo responsable del diseño y operación de la política cultural a nivel estatal; el segundo es un órgano consultivo y representativo de la comunidad cultural. ${ }^{5}$

El estado de Jalisco cuenta con una institucionalidad cultural normativa compuesta por varias leyes y reglamentos que tienen un alcance estatal por lo que sus municipios están obligados a atenderlas. Con referencia a los municipios, por ejemplo la Ley de Fomento a la Cultura en su artículo 7 les otorga a los gobiernos municipales las siguientes competencias:

1. Establecer las directrices municipales en materia de cultura, previa consulta a la comunidad cultural del municipio;

2. Procurar la creación de una dependencia municipal que tenga como funciones únicas las de llevar a la práctica los programas y acciones contenidas en el Programa Municipal de Cultura;

3. Celebrar los convenios necesarios con las instancias públicas estatales y federales, así como con las personas físicas o jurídicas de carácter privado, para la adecuada coordinación de las actividades culturales del municipio;

4. Elaborar y ejecutar programas para el desarrollo de las actividades culturales dentro del territorio municipal;

5. Fomentar la integración de organismos privados y sociales de promoción y divulgación de la cultura;

6. Fomentar la investigación de las manifestaciones culturales propias del municipio, sus ferias, tradiciones y costumbres;

7. Expedir los reglamentos en el ámbito de su competencia que normen la actividad cultural en el territorio municipal;

8. Otorgar premios, reconocimientos o estímulos a los individuos, organizaciones e instituciones públicas o privadas que se hayan destacado en

cos de iniciación al arte así como la conformación y fortalecimiento de grupos artísticos municipales; 3) Animación cultural, apoyando a los municipios a realizar presentaciones artísticas y facilitando la movilidad de los grupos artísticos municipales entre las regiones ; y 4) Capacitación cultural, que buscó el fortalecimiento competencias en gestión cultural de a través de cursos y talleres dirigidos a los agentes culturales municipales. 4 En esta investigación han colaborado en la recolección de datos Luis Gabriel Hernández Valencia, María Guadalupe Orozco Heredia, Genaro Baltazar Chávez Quintero, María del Carmen Valenzuela Gómez, Carlos Miguel Ochoa Oroz, Mónica Urrea Triana, Nicolás López Silva, Isabel Cuevas y Pilar Posada Rodríguez.

5 A pesar de la potencialidad que tiene un órgano como el CECA para facilitar la participación ciudadana, en realidad sus alcances son muy limitados, ya que no funge como un órgano consultivo regulador, sino más bien centra sus actividades en hacer algunas "observaciones" a los responsables de la política cultural pública (municipal y estatal) y administrar el Fondo Estatal para la Cultura y las Artes. 
la creación, promoción, preservación, difusión e investigación de la cultura en el ámbito de su jurisdicción;

9. Promover, en el ámbito de su competencia, las modalidades de descuento, pago de medio boleto o ingreso gratuito un día por semana a las representaciones teatrales, cinematográficas, ópera, ballet, danza y demás espectáculos públicos de carácter artístico o cultural;

10. Estimular la integración de Consejos Municipales para el Fomento de la Cultura, con la participación de la comunidad cultural, y los sectores sociales, privado y público;

11. Elaborar y mantener actualizado el registro y el directorio de las personas físicas o jurídicas que se dediquen al arte, tanto en la creación, fomento, apoyo, promoción como que dispongan de espacios apropiados para desarrollar actividades culturales en el municipio;

12. Elaborar y mantener actualizado un inventario de los espacios públicos con que cuenta el municipio para la realización de actividades culturales y artísticas;

13. Impulsar y proyectar en el ámbito estatal, nacional e internacional, en la medida de sus posibilidades, a los artistas municipales más destacados;

14. Procurar la creación de bibliotecas, hemerotecas, casas municipales de la cultura, museos, auditorios, teatros y centros culturales, así como procurar la ampliación, mantenimiento y mejoras físicas y tecnológicas; y

15. Conocer, analizar y resolver las solicitudes o peticiones que presenten personas físicas o jurídicas dedicadas a las actividades culturales, para la utilización de los espacios públicos con que cuenta el municipio.

Como se puede observar, la ley estatal le da competencias a los municipios para que puedan diseñar y operar políticas culturales normativas y de intervención directa, sin embargo no obliga o da una guía de las formas de institucionalidad orgánica que deben tener los municipios. Así, los gobiernos locales definen sus formas organizacionales de acuerdo a sus necesidades, trayectorias y prioridades. En términos generales podemos encontrar 6 tipos:

- Secretaría de cultura: Es una organización de primer nivel cuya estructura interna tiene tres subniveles (dirección general, dirección, subdirección), los cuales agrupan programas, proyectos, espacios culturales que a la vez cada uno cuenta con una programación cultural.

- Organismo público descentralizado: dependientes del presidente municipal, esta forma de organización (que toman la denominación de instituto), cuenta con programas y espacios culturales que a su vez tienen programan actividades. - Dirección de cultura: Son dependencias que forman parte del gabinete de primer nivel, regularmente cuentan con subdirecciones y responsables de espacios culturales (museos, casas de cultura, galerías, teatros, etc.). En algunos casos estas direcciones atienden también otras funciones por lo que se pueden encontrar combinaciones como "educación y cultura", "cultura y turismo" o "cultura y comunicación".

- Dirección dependiente de una dirección general: Son dependencias de segundo nivel. Regularmente están adscritas a direcciones de "desarrollo social", "Desarrollo humano", etc.

- Casa de Cultura: El presidente designa un director de la casa de cultura, la cual es la institución responsable de los servicios culturales en el municipio.

- Comisión edilicia: Un regidor se hace cargo de las actividades culturales, en 
algunas ocasiones apoyado por un pequeño grupo de personas, entre las que se podrían encontrar algún responsable de la casa de cultura.

Independientemente de sus formas de institucionalidad cultural, en los municipios de Jalisco hay un gran vacío en términos normativos en la caracterización y asignación de los responsables de las dependencias culturales gubernamentales, por lo que la incorporación o cese del trabajador cultural responde más a compromisos de los presidentes electos que a razones de planeación estratégica de los municipios o a reglamentos o normativas locales o estatales.

Como en gran parte de los municipios en Latinoamérica, en Jalisco los responsables de la acción cultural gubernamental han aprendido (y aprehendido) desde la misma práctica. Una buena parte del actuar de los agentes culturales se diseña y opera a partir de referentes empíricos, basados en experiencias propias de los gestores, experiencias que muy pocas veces son sistematizadas y reflexionadas.

\section{Perfiles de los responsables de la acción cultural municipal}

En la actualidad, se puede encontrar en los ayuntamientos de Jalisco una gran variedad de perfiles en cuanto a formación y experiencias en la gestión cultural. A partir de una investigación exploratoria ${ }^{6}$, los perfiles de los agentes culturales gubernamentales municipales son los siguientes:

En lo que se refiere al sexo hay equilibrio más o menos general, ya el $51 \%$ son mujeres y el $49 \%$ son hombres. La edad mínima registrada fue de 18 años y la máxima fue de 74 siendo el promedio 39 años. No obstante, el análisis estadístico nos brinda otros elementos para la discusión importantes. La media estadística es de 36 años, no obstante al analizar la distribución de los diferentes grupos de edad podemos encontrar los siguientes resultados: El primer grupo lo conforman agentes entre 18 y 25 años, el segundo entre 26 y 35, el tercero entre los 36 y 47 y el último entre los 48 y 74 años.

\begin{tabular}{|c|c|c|}
\hline Grupos & Personas & Porcentaje \\
\hline 18-26 años & 43 & $29 \%$ \\
\hline $27-35$ años & 33 & $22 \%$ \\
\hline 36-47 años & 39 & $26 \%$ \\
\hline $48-74$ años & 33 & $22 \%$ \\
\hline Total & 148 & $100 \%$ \\
\hline
\end{tabular}

Esta información nos indica, que la mitad de los agentes culturales gubernamentales de Jalisco tiene una edad entre 18 y 35 años. En lo que se refiere a la escolaridad, el 54\% su último grado fue el de licenciatura, $19 \%$ bachillerato, $5 \%$ secundaria, $8 \%$ una carrera técnica, $3 \%$ primaria, $10 \%$ maestría y sólo el $1 \%$ doctorado. Esto nos indica que el $65 \%$ de los agentes al menos tiene una licenciatura.

De los agentes que tienen grado o una carrera técnica, el $33 \%$ lo tiene en el área de las ciencias sociales, el $14 \%$ artísticas, el $13 \%$ administrativas y diseño, el $8 \%$ en ciencias exactas e ingenierías, el $2 \%$ de ciencias de la salud, el $4 \%$ de las ciencias biológicas, sólo el $2 \%$ en ingenierías y $2 \%$ en otras áreas que no se especificaron en el cuestionario. Cabe

$6 \mathrm{El}$ instrumento aplicado fue un cuestionario mixto (preguntas abiertas y cerradas) en soporte de papel. Fue aplicado de manera aleatoria a 148 directores y/o regidores responsables de la acción cultural municipal de 90 (correspondientes al 70\%) municipios de Jalisco. El cuestionario fue aplicado a un año de inicio de la administración municipal. 


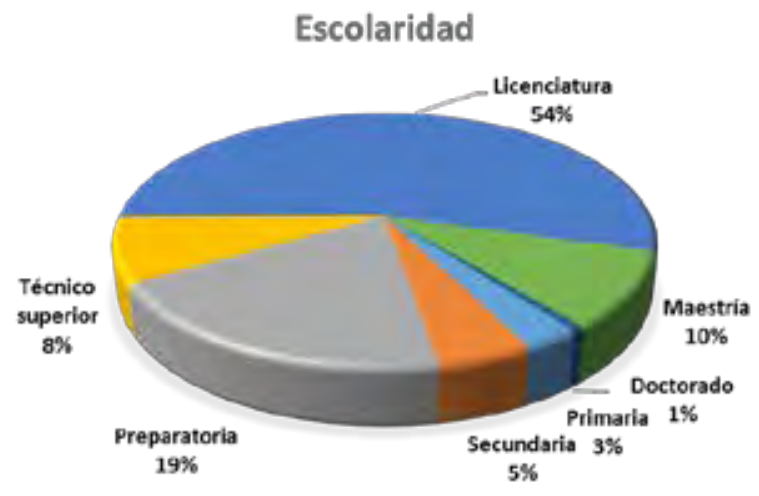

\section{Área disciplinar de estudios universitarios}

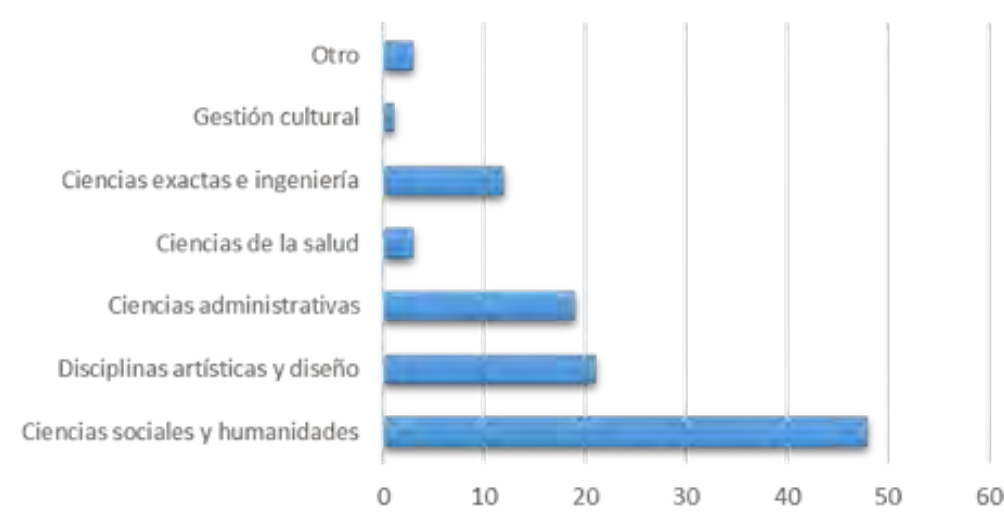

mencionar que sólo un $1 \%$ hizo referencia haber estudiado Gestión Cultural a nivel universitario. Sobre la experiencia en gestión cultural, se puede observar un predominio del 51\% de los agentes tiene un año o menos en el trabajo cultural, lo cual quiere decir, que al tomar la responsabilidad como trabajador de cultura de su municipio no tenían ninguna experiencia previa ${ }^{7}$. En cuestión numérica, le sigue otro grupo que dice tener entre dos y tres años $(15 \%)$ los cuales posiblemente fueron ratificados en su puesto con la nueva administración. El tercer grupo lo componen aquellos que dijeron tener entre 4 y 9 años (13\%) y sólo el $13 \%$ restante tiene entre 9 y 43 años. Esta información nos permite hacer un primer acercamiento para observar que en la mayoría $(85 \%)$ de los responsables de la gestión cultural en los municipios de Jalisco no tienen una experiencia en gestión cultural mayor a la de dos administraciones de gobierno municipal.

\begin{tabular}{|c|c|c|}
\hline Grupos & Personas & Porcentaje \\
\hline $0-1$ año & 76 & $51 \%$ \\
\hline 2-3 años & 22 & $15 \%$ \\
\hline 4-8 años & 19 & $13 \%$ \\
\hline 9-43 años & 19 & $13 \%$ \\
\hline Total & 148 & $100 \%$ \\
\hline
\end{tabular}

7 Esto debido al tiempo en que se aplicó el cuestionario. 


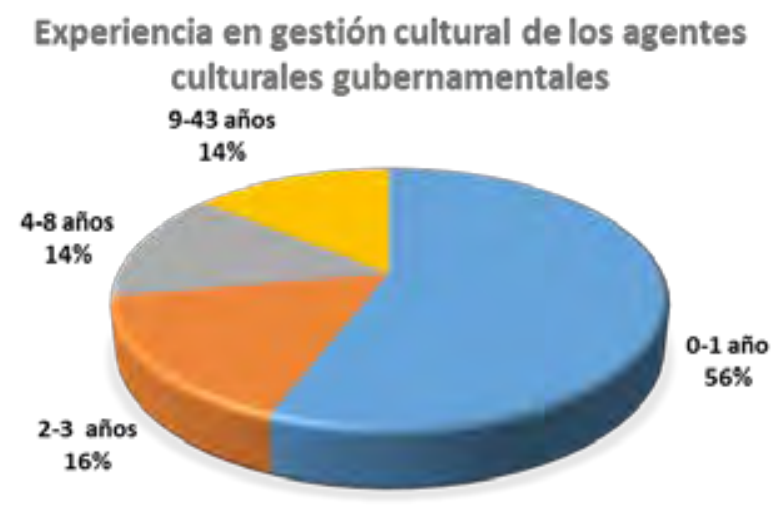

Sobre su dedicación a la gestión cultural municipal, se les preguntó si su labor como gestor era su principal fuente de ingresos económicos o no, así se observó que el 33\% tiene como única fuente de ingresos la gestión cultural municipal, el $41 \%$ la refirió como una fuente de ingresos importante pero no la única, mientras que el 19\% mencionó que es una fuente complementaria. Sólo el 7\% están haciendo el trabajo cultural en el ayuntamiento de manera voluntaria.

\section{La GC como fuente de ingresos}

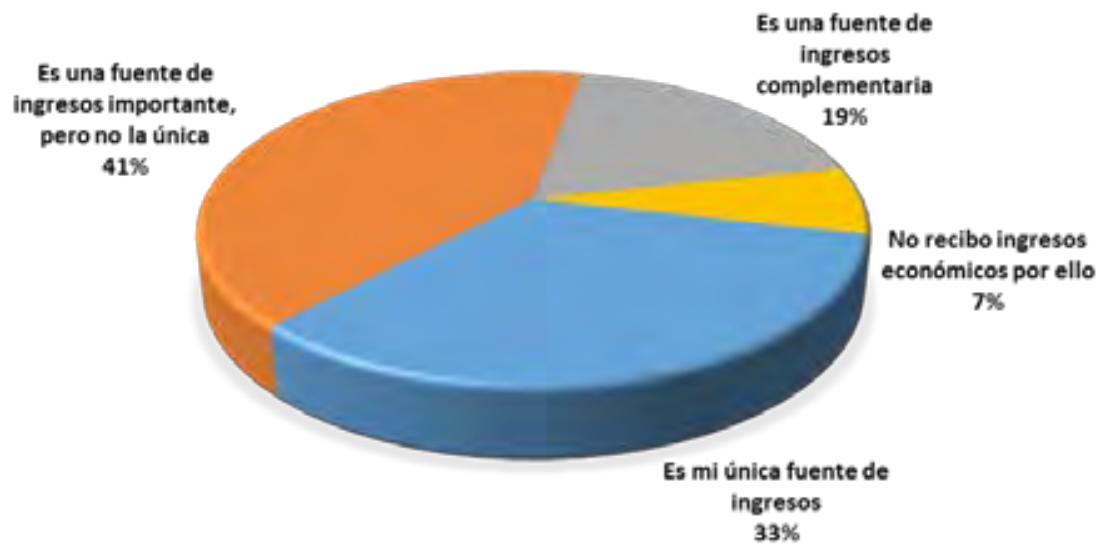

En lo referente a las competencias en gestión cultural que los agentes dicen tener, se observó que las cuatro competencias más recurrentes son: Organización y operación de actividades culturales, difusión de actividades culturales, diseño de proyectos culturales y diagnóstico cultural, en cambio las tres competencias menos mencionadas fueron diseño de políticas culturales, conocimientos de gestión cultural y evaluación de proyectos culturales.

El 57\% de los agentes no han participado en ningún programa de capacitación antes o durante su función. No obstante, las formas en que han adquirido dichas competencias han sido de manera autodidacta. De los tipos de capacitación a los que sí han participado, han sido primordialmente curso, talleres y conferencias. En menor medida fueron diplomados, licenciatura u especialidad. No se registró ningún agente con posgrado (maestría o doctorado) en gestión cultural. 
No obstante las competencias que dicen tener, el $99 \%$ está interesado en participar en algún programa de formación para poder desarrollar sus competencias laborales principalmente las siguientes cuatro: Diseñar proyectos culturales, diseñar políticas culturales y el manejo de una metodología de la gestión cultural.

La información presentada hasta ahora, nos da una visión general del perfil de los agentes culturales gubernamentales. A partir de la información que se proporcionó en el cuestionario y de la etnografía, podríamos tener acercamiento a una caracterización preliminar de tipos de agentes municipales. En ese sentido, podríamos identificar cuatro tipos ${ }^{8}$ :

- Un primer grupo está compuesto por jóvenes entre 18 y 30 años, generalmente egresados de una licenciatura y que tienen entre 1 y 3 años de experiencia en la gestión cultural. La mayoría de ellos ha participado en algún programa de capacitación en gestión cultural. Esto nos indica, que este tipo de agentes tienen relativamente poca experiencia laboral al momento de que se les asigna el cargo y sus competencias en gestión cultural las desarrollan durante el ejercicio de su función.

- El segundo grupo está conformado por personas entre 32 y 44 años de edad. La mitad de ellos cuentan ya con una licenciatura y la otra mitad estudiaron bachillerato o una carrera técnica. Este tipo de agentes ha participado entre 4 y 8 años en la promoción y difusión cultural en su municipio. La mayoría de ellos no ha participado en programas de capacitación cultural, pero ha desarrollado sus competencias de manera autodidacta.

- El tercer grupo está compuesto por personas entre 38 y 60 años de edad. Han participado como promotores culturales en sus municipios de 10 a 30 años. La mayoría de ellos han estudiado al menos una licenciatura y participado programas de capacitación cultural.

- Un último grupo está compuesto por personas que al tomar su cargo fue su primer acercamiento con la gestión cultural. Es bastante heterogéneo tanto en las edades (entre los 22 y los 48 años) y su nivel escolar (Principalmente bachillerato y licenciatura). Otra cuestión que los caracteriza es que no han participado en ningún programa de capacitación cultural.

Esta radiografía de los perfiles de directores y regidores de cultura de los municipios de Jalisco, nos da algunos elementos para entender por qué gran parte de la gestión cultural municipal se centra sólo en la programación de actividades. A continuación se analizarán las acciones culturales recurrentes en los municipios a la luz de las propuestas que se plantean en la Agenta21 de la cultura local.

\section{La gestión cultural municipal en Jalisco ante la Agenda21 de la cultura local}

En la actualidad y a nivel internacional, uno de los documentos que sirven como guía para el desarrollo de políticas culturales a nivel municipal es la Agenda 21 de la cultura. Este documento fue aprobado el 8 de mayo de 2004 en el marco del Foro Universal de las Culturas en la ciudad de Barcelona, España. Su propósito es servir como "documento orientador de las políticas públicas de cultura y como contribución al desarrollo cultural de la humanidad" (Ciudades y Gobiernos Locales Unidos, 2004:53).

En términos generales son tres elementos los que fundamentan conceptualmente la Agenda 21 de la cultura. El primero es la visión del desarrollo sostenible como modelo de acción de largo alcance para el futuro. No obstante, existen cuatro pilares fundamentales

8 La tipología se esbozó con fines analíticos y debe verse en términos relacionales y de carácter exploratorio, el cual, puede dar elementos para un estudio posterior de mayor profundidad. 
para lograr la sustentabilidad: a) Vitalidad cultural, que se basa en el bienestar, la creatividad, la diversidad cultural y la innovación; b) equidad social, que implica la justicia, compromiso ciudadano, calidad de vida y cohesión social; c) responsabilidad ambiental, que busca un balance ecológico; y d) viabilidad económica, que se ve reflejado en una prosperidad material (Hawkes, 2001: 25).

El segundo elemento es la centralidad de la cultura como un eje estratégico en el desarrollo sostenible, ya que si en una sociedad se desintegra su cultura, también lo hará todo lo demás, por lo que toda acción que pretenda el desarrollo debe contemplar su requerimiento cultural (Ibíd:: 12). Así pues, una sociedad sana depende, de una actividad cultural viva e influyente entre las comunidades y al interior de ella; la sostenibilidad sólo puede lograrse cuando esta llega a ser abrazada con entusiasmo como parte de nuestra cultura (Ibíd.: 25).

El último elemento tiene que ver con la importancia del gobierno local. A diferencia de otros documentos orientadores internacionales ${ }^{9}$, la Agenda21 de la cultura especifica su ámbito de acción en los gobiernos locales, que en el caso de México se podría definir como el Municipio. Desde esta posición, se observa al municipio como la unidad básica desde donde se pueden hacer cambios significativos en las dinámicas cultuales de las sociedades a partir de acciones gubernamentales en las que participen activamente la ciudadanía. Así pues, bajo estos fundamentos la Agenda21 de la cultura define los siguientes principios rectores ${ }^{10}$ :

1. La diversidad cultural es el principal patrimonio de la humanidad. Es el producto de miles de años de historia, fruto de la contribución colectiva de todos los pueblos, a través de sus lenguas, imaginarios, tecnologías, prácticas y creaciones. La cultura adopta formas distintas, que siempre responden a modelos dinámicos de relación entre sociedades y territorios, por lo tanto la diversidad cultural constituye uno de los elementos esenciales de transformación de la realidad urbana y social.

2. La cultura, como el medio ambiente, es un bien común de la humanidad. Debido a la situación actual, la diversidad cultural en el mundo se halla en peligro debido a una mundialización estandarizadora y excluyente. Sin embargo, como afirma la UNESCO, la diversidad cultural es tan necesaria para el género humano como la biodiversidad para los seres vivos.

3. Los gobiernos locales reconocen que los derechos culturales forman parte indisociable de los derechos humanos En tal sentido, se ratifica que la libertad cultural de los individuos y las comunidades resulta condición esencial de la democracia.

4. Los gobiernos locales se constituyen como agentes mundiales de primer orden, en tanto defensores y promotores del avance de los derechos humanos. Además trabajan conjuntamente en red, intercambiando prácticas, experiencias y coordinando sus acciones.

5. El desarrollo cultural se apoya en la multiplicidad de los agentes sociales. Los principios de un buen gobierno incluyen la transparencia informativa y la participación ciudadana en la concepción de las políticas culturales, en los procesos de toma de decisiones y en la evaluación de programas y proyectos. 6. La indispensable necesidad de crear las condiciones para la paz debe caminar junto a las estrategias de desarrollo cultural. La guerra, el terrorismo,

9 Como la Declaración de los Principios de la Cooperación Cultural Internacional (Unesco, 1966), la Declaración de México sobre las Políticas Culturales (Unesco, 1982), la Declaración Universal sobre la Diversidad Cultural (Unesco, 2001), la Carta Cultural Iberoamericana (Cumbre Iberoamericana, 2006), etc.

10 Los principios como los compromisos de la Agenda21 de la cultura local presentados aquí, han sido resumidos con propósitos de expositivos acorde al interés de esta ponencia. 
la opresión y la discriminación son expresiones de intolerancia que deben ser condenadas y erradicadas.

7. Las ciudades y los espacios locales son un marco privilegiado de la elaboración cultural en constante evolución y constituyen los ámbitos de la diversidad creativa, donde la perspectiva del encuentro de todo aquello que es diferente y distinto (procedencias, visiones, edades, géneros, etnias y clases sociales) hace posible el desarrollo humano integral.

8. La convivencia en las ciudades implica un acuerdo de responsabilidad conjunta entre ciudadanía, sociedad civil y gobiernos locales. El ordenamiento jurídico resulta fundamental pero no puede ser la única regulación de la convivencia en las ciudades.

9. El patrimonio cultural, tangible e intangible, es el testimonio de la creatividad humana y el substrato de la identidad de los pueblos. La vida cultural contiene simultáneamente la riqueza de poder apreciar y atesorar tradiciones de los pueblos, con la oportunidad de permitir la creación y la innovación de sus propias formas.

10. Las políticas culturales constituyen un factor esencial en el desarrollo sostenible de de las ciudades en el aspecto humano, económico, político y social. No obstante la calidad del desarrollo local requiere la imbricación entre las políticas culturales y las demás políticas públicas -sociales, económicas, educativas, ambientales y urbanísticas.

11. Las políticas culturales deben encontrar un punto de equilibrio entre interés público y privado, vocación pública e institucionalización de la cultura. Una excesiva institucionalización, o la excesiva prevalencia del mercado como único asignador de recursos culturales, comporta riesgos y obstaculiza el desarrollo dinámico de los sistemas culturales. La iniciativa autónoma de los ciudadanos, individualmente o reunidos en entidades y movimientos sociales, es la base de la libertad cultural.

12. La adecuada valoración económica de la creación y difusión de los bienes culturales -de carácter aficionado o profesional, artesanal o industrial, individual y colectivo- es un derecho democrático de los pueblos a afirmar sus identidades en las relaciones entre las culturas. Es necesario destacar la importancia de la cultura como factor de generación de riqueza y desarrollo económico.

13. El acceso al universo cultural y simbólico constituye un elemento fundamental de formación de la sensibilidad, la expresividad, la convivencia y la construcción de ciudadanía.

14. La apropiación de la información y su transformación en conocimiento por parte de los ciudadanos es un acto cultural. Por lo tanto, el acceso sin distinciones a los medios de expresión, tecnológicos y de comunicación fortalece la dinámica de las culturas locales.

15. El trabajo es uno de los principales ámbitos de la creatividad humana. Su dimensión cultural debe ser reconocida y desarrollada. La organización del trabajo y la implicación de las empresas en la ciudad o el territorio deben respetar esta dimensión como uno de los elementos fundamentales de la dignidad humana y del desarrollo sostenible

16. Los espacios públicos son bienes colectivos que pertenecen a todos los ciudadanos. Ningún individuo o grupo puede verse privado de su libre utilización, dentro del respeto a las normas adoptadas en cada ciudad. 
A partir de la información generada en la investigación ${ }^{11}$, podemos hacer una serie de acercamientos a los avances y retos que tendrían que atender los municipios en los próximos años. Para realizar este análisis reorganizar los compromisos que especifica la Agenda21 de la cultura a partir de seis dimensiones de acción:

\section{Fomento y preservación de la diversidad}

En términos generales, las acciones culturales de los municipios de Jalisco consideran la importancia de la diversidad cultural, sin embargo esta es definida sobre todo en términos de patrimonio cultural (regularmente de bienes tradicionales e históricos). Se argumenta, por ejemplo, la importancia de las culturas populares e indígenas vistas estas como ese patrimonio cultural que es una herencia, en algunas ocasiones particulares de los municipios (que le es significativo sólo al municipio) y en otras es vista como herencia de una "cultura nacional" apropiando el discurso oficial del Estado. ${ }^{12}$ De las principales acciones que se realizan en este ámbito se encuentran:

- Creación, consolidación y promoción de "grupos de danza folclórica" donde se reinterpreta una cultura popular generalmente del pasado. En algunos casos la reinvención de tradiciones locales "ya perdidas" o "a punto de desaparecer". - Elaboración y publicación de las historias de los municipios.

- Generación de recorridos turísticos.

- Rehabilitación y restauración de patrimonio cultural mueble e inmueble, en particular el religioso.

Con miras de aprovechar lo que los municipios hacen actualmente, los retos que enfrentan los gobiernos locales con respecto a la primera dimensión son:

- Dar un cambio de modelo que vaya de la preservación y difusión del patrimonio cultural local, a la gestión de dicho patrimonio. Esto implicaría generar acciones integrales que contemplen la investigación, conservación, preservación y promoción de los patrimonios tangibles e intangibles; pero no como mercancías listas para el consumo turístico, sino como elementos de significación y resignificación de las comunidades y sus historias.

- Equilibrio entre el turismo para visitantes y locales, ya que una buena parte de las acciones de promoción cultural van encaminadas a los turistas pero muy pocas veces se integra a los habitantes de las localidades para el deleite y recreación.

- Reconocimiento de la diversidad cultural en los mismos municipios, no sólo en términos de grupos étnicos, sino también de grupos sociales más amplios cuya identidad y prácticas culturales se diferencien por su género, edad, religión y pertenencia territorial.

- Expedir reglamentos y lineamientos normativos municipales que protejan

11 Esta información se recuperó a partir de varias técnicas: Trabajo etnográfico con observación directa y entrevistas in situ en los espacios de trabajo de 8 municipios; revisión documental de informes y proyectos de los agentes culturales así como fuentes hemerográficas y bibliográficas; entrevistas abiertas; y la investigación acción participativa (realización de talleres de diagnóstico) y entrevistas grupales con agentes culturales (cerca del 80\% de los municipios del estado) en el marco del Encuentro Estatal de Regidores y Directores de Cultura de Jalisco realizado en cuatro ocasiones: 2009, 2010, 2011 y 2012.

12 Es importante esta cuestión, ya que algunos bienes patrimoniales no se podrían considerar como patrimonio cultural local, sin embargo, es apropiado y resignificado por los agentes culturales como suyo, aunque no esté en su territorio; es suyo, por el hecho de identificarse como mexicanos. 
el patrimonio cultural local y que fomente la convivencia entre los diversos grupos y sus identidades cultuales.

\section{Participación ciudadana}

Estratégicamente hablando, es la pieza más importante para el fortalecimiento de la democracia y un ejercicio de ciudadanía, pero, sobre todo, para la continuidad del trabajo cultural que se realiza en los municipios. De lo que actualmente se realiza es:

- La acción cultural se diseña de acuerdo a consideraciones de los agentes municipales y en su mayoría poco sistemático. Se centra más en la programación de "eventos artísticos y culturales" que en el diseño de planes estratégicos basados en diagnósticos participativos.

- En algunos municipios existen consejos ciudadanos, pero funcionan como pequeños comités u asociaciones que reciben recursos del fideicomiso "Programa de Desarrollo Cultural Municipal", por lo que su actuar va encaminada a la organización de actividades culturales de manera paralela a las direcciones de cultura.

En ese sentido, los retos a tomar en cuenta en esta dimensión son:

- Fortalecer los consejos ciudadanos con una función no sólo consultiva, sino resolutiva, de tal manera que permitiese la participación ciudadana para la toma de decisiones y seguimiento de la acción cultural municipal. Esto, además de pertinencia, sería una fuente de legitimación de la política cultural municipal. - Llama la atención que en gran parte de los municipios hay uno o varios "grupos de danza" (regularmente folclórica) que además de ser grupos artísticos ciudadano reconocidos por las comunidades, también suelen ser semilleros de promotores culturales, ya que varios de sus integrantes comenzaron a hacer gestión cultural en el grupo y posteriormente lo aplican a otros ámbitos e incluso en alunas ocasiones suelen llegar a ser directores de cultura de sus municipios. En ese sentido, una propuesta sería fortalecer las competencias de gestión de este tipo de grupos para que sigan formando en la práctica a gestores culturales independientes (pero ahora con conciencia y mejor preparación).

- Realizar modificaciones a la Ley Estatal de Fomento a la Cultura para que contemple, una institucionalidad cultural orgánica básica para los municipios de Jalisco en la que se considere la participación de la ciudadanía en el diseño y seguimiento de la política cultural municipal creando la figura de Consejo Ciudadano Municipal de Cultura para esos fines.

\section{Articulación de políticas públicas}

Dado que la cultura es la columna vertebral de la sociedad, es importante que toda política cultural esté articulada con otras políticas públicas para permitir un mayor alcance de la acción cultural. En este sentido, en algunos municipios se ha intentado contemplar el "ámbito cultural" como parte de los trabajos que realizan los comités de planeación para el desarrollo municipal (COPLADEMUN). El gran problema que se observa, es que la acción cultural que se suele proponer en esas mesas tiene una visión de cultura como producto y no como proceso, privilegiando actividades culturales tales como talleres, festivales y algunas exposiciones que no se articulan con los objetivos y metas de desarrollo de los municipios en otros ámbitos (como el educativo, seguridad pública, infraestructura municipal o incluso 
el de salud), por lo que se ve a la cultura más como un adorno y no como una herramienta de comunicación de contenidos y toma de conciencia.

En ese sentido, uno de los grandes retos es la generación de planes de desarrollo cultural municipal integrales con la participación de agentes culturales cuya visión de la cultura sea amplia y articuladora de otros ámbitos de la política púbica

\section{Apropiación y acceso de la infraestructura, los bienes y servicios culturales}

Esta dimensión está relacionada con el derecho al acceso a la cultura, al menos como actualmente se entiende en nuestra constitución. De las acciones que se realizan actualmente son:

- Festivales culturales en plazas públicas de las cabeceras municipales y de algunas delegaciones.

- Creación y rehabilitación de espacios culturales, regularmente casas de cultura o museos municipales.

- Implementación de talleres artísticos regularmente en las casas de la ctlura municipal.

- Algunos museos ya cuentan con un programa de actividades que les permite la animación dentro de su institución.

- Políticas centradas en la producción y circulación.

De los retos a considerar en esta dimensión se encuentran las siguientes acciones:

- Definición de los perfiles deseados en los diferentes niveles de agentes culturales municipales: Regidor, director de cultura, coordinador de programa o responsable de espacio cultural, promotor cultural, animador, etc.

- Actualmente la gran mayoría de la acción cultural municipal está enfocada al a fase de producción y consumo cultural, sin embargo hace falta considerar la circulación y el intercambio como un interés de la política cultural local, de tal manera que se puedan generar diversos circuitos culturales que articulen producciones, públicos y espacios diferenciados a los diferentes grupos sociales de acuerdo a sus intereses, permitiendo así que los grupos artísticos puedan enfrentar las condiciones desiguales que les impone la cultura de masas.

- Formación de públicos que permita generar puentes entre creadores, productores, medios y públicos.

- Generación y/o adecuación de algunos reglamentos municipales para asegurar una política de accesibilidad de los servicios culturales a las personas con alguna discapacidad visual, auditiva, motora y/o mental.

- Generar una programación de actividades culturales diversificada y equitativa que atienda a las necesidades, gustos y capacidades de los diversos grupos demográficos: niños, jóvenes, adultos y ancianos.

- Implementación de acciones que permitan el uso de los espacios públicos para la realización de actividades culturales que fomenten la convivencia, la recreación y la integración de diversos grupos sociales.

- Apertura de los espacios culturales institucionales (museos, galerías, casas de cultura, auditorios, teatros, etc.) para que la programación anual de dichas instituciones se dé a partir de un concurso público de propuestas presentadas y evaluadas por la propia comunidad cultural. 


\section{Empresas culturales}

En esta dimensión, la inmensa mayoría de los municipios no realizan actividades encaminadas a potencializar a las empresas culturales. El único esfuerzo que se ha realizado en este sentido, ha sido desde el gobierno estatal, a través de la Secretaría de Cultura y la Secretaría de Promoción Económica a través del Fondo Jalisco de Fomento Empresarial (FOJAL) se conformó el Fondo para la Cultura y las Artes (FOCULTA) que apoya con capacitación y financiamiento crediticio a empresas creativas de Jalisco. Bajo un modelo de incubadora de negocios, han apoyado a cerca de144 entre el 2010 y el 2012. (FOJAL, 2012)

Aunque este programa de financiamiento está teniendo buenos resultados, debería ir acompañado de algunas disposiciones fiscales y administrativas en los tres niveles de gobierno. A nivel nacional, se debe estimular a las micro y pequeñas empresas, así como profesionistas de la cultura con una reducción significativa de impuestos. A nivel estatal y municipal deben de simplificar los trámites y dar todas las facilidades para el establecimiento y mantenimiento de empresas que tengan como base de negocio la realización y comercialización de bienes y servicios culturales, ya que en muchas ocasiones en los padrones de licencia municipal pueden entrar en la misma categoría sacar un permiso para un centro nocturno de adultos que un centro cultural independiente.

\section{Cooperación e intercambio}

Esta última dimensión, está relacionada con la capacidad que tienen los gobiernos locales de crear vínculos entre sí a nivel nacional e internacional. Gran diversos municipios de Jalisco tienen como práctica común tener "ciudades hermanas", esto es, entre gobiernos locales se llevan a cabo convenios de intercambio y reciprocidad regularmente de índole "cultural" y de asistencialismo. Hay una preferencia por las ciudades extranjeras, regularmente de Estados Unidos, y muchas veces los municipios jaliscienses buscan donaciones de ambulancias, carros de bomberos etc., así como organizar muestras de la cultura local jalisciense en la ciudad hermana. Regionalmente, suele haber intercambios "culturales" entre los municipios consistentes en recibir y enviar los grupos artísticos locales, regularmente en las fechas en que se realiza la feria municipal. Regularmente este tipo de intercambios se da a partir de la empatía entre regidores y directores delos municipios y no de manera formal a través de un convenio.

Una de las apuestas, podría ser el fortalecimiento del intercambio regional, nacional e internacional con otras ciudades pero a partir de proyectos de coproducción de tal manera que los gobiernos jaliscienses sean más proactivos y que los "hermanamientos" sean vistos como una oportunidad de trabajar conjuntamente con otros gobiernos locales y no tanto como una oportunidad de recibir algo de afuera.

\section{Conclusiones}

Los gobiernos locales generan acciones culturales que repercuten en las comunidades, sin embargo, dichas acciones se diseñan y operan a partir de ciertos vacíos e inconsistencias de la institucionalidad cultural normativa y orgánica, por lo que la gestión cultural municipal va a estar determinada en gran parte por los perfiles y visiones de la cultura que tengan los agentes culturales gubernamentales.

Hay una gran movilidad en la ocupación de los puestos de las direcciones de cultura y de los espacios culturales municipales, ya que el acceso y permanencia se da a partir de una fidelidad política partidista al candidato que gana la presidencia municipal y no tanto a la experiencia de los agentes en el ámbito cultural. Se puede decir que casi el 80\% de los agentes culturales municipales cambian cuando cambia la administración, y el 56\% de estos toma la responsabilidad de la política cultural local sin ninguna experiencia previa en el 
campo de la gestión cultural. Esto en parte se debe a la carencia de lineamientos normativos que establezcan funciones y perfiles mínimos requeridos para ocupar el puesto, criterios de evaluación y un sistema de seguimiento que permita sistematizar experiencias y analizar impactos y alcances de las políticas culturales locales.

No obstante, los gobiernos locales como parte de su cotidianidad organizan actividades culturales, sobre todo de carácter artístico y patrimonialista, sin embargo gran parte de dichas acciones son efímeras, realizadas a partir de la ocurrencia y sin un diagnóstico previo que permita construir planes estratégicos a corto, mediano y largo plazo.

En Jalisco, actualmente se cuenta con una infraestructura cultural aceptable en los municipios, que requieren de normatividades que los conserve y de una programación cultural que requiere de la participación ciudadana para la pertinencia y persistencia de los contenidos y las formas de la acción cultural a nivel local, sobre todo considerando la gran centralización que existe en el estado (la Zona Metropolitana de Guadalajara concentra buena parte del presupuesto y de los servicios culturales).

Si bien es cierto que las modificaciones a la Ley de Fomento a la Cultura no dependen de los gobiernos municipales, hay algo que éstos sí pueden hacer, y es apostar por fortalecer las capacidades en gestión cultural de sus agentes municipales, generar lineamientos normativos que propicien la participación ciudadana en el desarrollo de la política pública en materia de cultura (articulada con las otras políticas públicas) que gestione el patrimonio cultural tangible e intangible local; genere circuitos culturales que beneficien a la producción y consumo cultural local; forme públicos a partir de una oferta pertinente a los diversos grupos sociales y participando activamente en proyectos de coproducción con municipios que para solucionar problemáticas similares a partir de la complementariedad.

\section{Bibliografía}

Castro de la Mora, Omar (2011), La política cultural en Jalisco. Guadalajara: Consejo Estatal para la Cultura y las Artes, Secretaría de Cultura de Jalisco.

Ciudades y Gobiernos Locales Unidos (2004), Agenda 21 de la cultura, Barcelona: Ayuntamiento de Barcelona.

Flores Cruz, Cipriano (1985), "Municipio: gobierno de la comunidad" en Gaceta Mexicana de Administración Pública Estatal y Municipal, Número 16-17. p. 65-68

FOJAL (2012), Ficha informativa de FOCULTA- FOJAL. [Fecha de consulta: 12 de febrero de 2013]. Disponible en: http://www1.jalisco.gob.mx/wps/portal/organismos/fojal/ moduloatencion/!ut/p/c5/04_SB8K8xLLM9MSSzPy8xBz9CP0os3gzb2djr1AXEwOLoABTA0_nkCATA0cfR0NvI6B8pFm8T4iPh7FXkJGBf5iXs4FRmI-5pbllsKGBvzEB3eEg-_DrB8kb4ACOBhB5uA0Gzm6OBp4WgWFeIb6-Hq6BZmjyaC6wNNH388jPTdUvyI0wyPTUdQQAJpfzCA!!/dl3/d3/L2dBISEvZ0FBIS9nQSEh/

Garretón M, Manuel Antonio (2008). Las políticas culturales en los gobiernos democráticos en Chile, Políticas culturais na Ibero-América, 75-118.

Gutiérrez Salazar, Elías Sergio (1985), "Comunidad básica, municipio y centralismo" en Gaceta Mexicana de Administración Pública Estatal y Municipal, Número 16-17. Pp 57-59.

Hawkes, Jon (2001), The fourth pillar of sustainability. Culture's essential role in public planning, Victoria: Cultural Development Network.

Instituto Nacional de Estadística, Geografía e Informática (2011), Censo de Población 2010, Jalisco. México: INEGI.

Vicencio Tovar, Abel (1986), "El municipio: célula política y ciudadana” en Cuadernos del Instituto de Investigaciones Jurídicas, Año I, número 2. Mayo - agosto, UNAM. pp. 799-807.

Riva Palacio López, Antonio (1985), "El municipio como institución básica de la estructura política mexicana" en Gaceta Mexicana de Administración Pública Estatal y Municipal, Número 16-17. pp. 37-39.

Secretaría de Cultura de Jalisco (2009), Conclusiones del Primer Encuentro Estatal con Regidores y Directores de Cultura de Jalisco, Puerto Vallarta: Inédito. 
(2010), Conclusiones del Segundo Encuentro Estatal con Regidores y Directores de Cultura de Jalisco, Guadalajara: Inédito.

(2011), Conclusiones del Tercer Encuentro Estatal con Regidores y Directores de Cultura de Jalisco, Puerto Vallarta: Inédito.

(2012a), Conclusiones del Cuarto Encuentro Estatal con Regidores y Directores de Cultura de Jalisco, Puerto Vallarta: Inédito.

(2012b), Informe de actividades 2007 - 2012. Guadalajara: Gobierno del Estado de Jalisco.

\section{3}

